International Mathematical Forum, Vol. 9, 2014, no. 32, 1553 - 1561

HIKARI Ltd, www.m-hikari.com

http://dx.doi.org/10.12988/imf.2014.48148

\title{
Some Fixed Point Results for $w$-distances
}

\author{
M. Azhini and S. Z. Rasouli Keneti
}

Department of Mathematics, Science and Research Branch

Islamic Azad University, Tehran, Iran.

Copyright (c) 2014 M. Azhini and S. Z. Rasouli Keneti. This is an open access article distributed under the Creative Commons Attribution License, which permits unrestricted use, distribution, and reproduction in any medium, provided the original work is properly cited.

\begin{abstract}
In this paper, by using $w$-distances we improve and extend [Harjani, K. Sadrangani, "Fixed point theorems for weakly contractive mappings in partially ordered sets" in Ninlinear Analysis 71 (2009) 3403-3410.
\end{abstract}

Mathematics Subject Classification: 47H10, 47H09

Keywords: Fixed point; partially ordered set; w-distance; nondecresing mapping; nonincreasing mapping

\section{Introduction}

In 1996, Kada, Suzuki and Takahashi [1],[2] introduced the concept of $w$ distance on a metric space. They elaborated, with the help of examples, that the concept of $w$-distance is general than that of metric on a nonempty set. They also proved a generalization of Caristi fixed point theorem employing the definition of $w$-distance on a complete metric space.

In this paper, we prove some results for weakly contractive mappings in partially ordered sets by considering the concept of $w$-distance our results improve and extend $[2]$.

Definition 1.1. Let $(X, d)$ be a metric space. Then a function $p: X \times X \longrightarrow$ $[0, \infty)$ is called a $w$-distance on $X$ if the followings are satisfied: 
(1) $p(x, z) \leq p(x, y)+p(y, z)$ for any $x, y, x \in X$.

(2) For any $x \in X, p(x,):. X \longrightarrow[0, \infty)$ is lower semicontinuous.

(3) For each $\varepsilon>0$, there exists $\delta>0$ such that $p(z, x) \leq \delta$ and $p(z, y) \leq \delta$ implies $d(x, y) \leq \varepsilon$.

Example 1.2. If $X=\left\{\frac{1}{n} \mid n \in \mathbb{N}\right\} \cup\{0\}$ for each $x, y \in X, d(x, y)=x+y$ if $x \neq y$ and $d(x, y)=0$ if $x=y$ is a metric on $X$ and $(X, d)$ is a complete metric space. Moreover, by defining $p(x, y)=y, p$ is a $w$-distance on $(X, d)$.

Example 1.3. Let $(X, d)$ be a metric space. Then a function $p: X \times X \longrightarrow$ $[0, \infty)$ defined by $p(x, y)=k$ for every $x, y \in X$ is a $w$-distance on $X$, where $k$ is a positive real number. But $p$ is not a metric since $p(x, x)=k \neq 0$ for any $x \in X$.

Example 1.4. [10] Let $(X, d)$ be a metric space and let $g$ be a continuous mapping from $X$ into itself. Then a function $p: X \times X \longrightarrow[0, \infty)$ defined by

$$
p(x, y)=\max \{d(g x, y), d(g x, g y)\}
$$

for every $x, y \in X$ is a $w$-distance on $X$.

Definition 1.5. Let $p$ be a $w$-distance on a metric space $(X, d)$. Suppose that $\Phi$ is the set of functions

$$
\phi:[0, \infty) \longrightarrow[0, \infty)
$$

where $\varphi$ is non-decreasing, continuous and $\varphi(\varepsilon)>0$ for each $\varepsilon>0$. Moreover, let $\Psi$ be the set of functions

$$
\psi:[0, \infty) \longrightarrow[0, \infty)
$$

where $\psi$ is non-decreasing, right continuous and $\psi(t)<t$ for all $t>0$.

Example 1.6. Let $\left\{a_{n}\right\}_{n=1}^{\infty}$ and $\left\{c_{n}\right\}_{n=1}^{\infty}$ are two non-negative sequences such that $\left\{a_{n}\right\}$ strictly decreasing, convergence to zero, and for each $n \in \mathbb{N}$,

$$
c_{n-1} a_{n}>a_{n+1} \quad \text { where } \quad 0<c_{n-1}<1
$$

define $\psi:[0, \infty) \longrightarrow[0, \infty)$ by $\psi(0)=0, \psi(t)=c_{n} t$, if $a_{n+1} \leq t<a_{n}$, $\psi(t)=c_{0} t$ if $t \geq a_{1}$, then $\psi$ is in $\Psi$. 
Lemma 1.7. [9] If $\psi \in \Psi$, then $\lim _{n} \psi^{n}(t)=0$ for each $t>0$; and if $\varphi \in \Phi$, $\left\{a_{n}\right\} \subseteq[0, \infty)$ and $\lim _{n} \varphi\left(a_{n}\right)=0$, then $\lim _{n} a_{n}=0$.

Lemma 1.8. [7] Let $(X, d)$ be a metric space and $p$ be a $w$-distance on $X$. If $\left\{x_{n}\right\}$ is a sequence in $X$ such that

$$
\lim _{n} p\left(x_{n}, x\right)=\lim _{n} p\left(x_{n}, y\right)=0,
$$

then $x=y$. In particular, if $p(z, x)=p(z, y)=0$ then $x=y$.

If $p(a, b)=p(b, a)=0$. Then by lemma $1.8, a=b$.

Lemma 1.9. [7] Let $p$ be a w-distance on a metric space $(X, d)$ and $\left\{x_{n}\right\}$ be a sequence in $X$ such that for each $\varepsilon>0$ there exists $N_{\varepsilon} \in \mathbb{N}$ such that $m>n>N_{\varepsilon}$ implies $p\left(x_{n}, x_{m}\right)<\varepsilon$ or

$$
\lim _{m, n} p\left(x_{n}, x_{m}\right)=0,
$$

then $\left\{x_{n}\right\}$ is a Cauchy sequence.

\section{Main Results}

Theorem 2.1. Let $(X, \leq)$ be a partially ordered set. Suppose that there exists a metric $d$ in $X$ such that $(X, d)$ is a complete metric space and let $p$ be a $w$ distance on $X$. Let $f: X \longrightarrow X$ be a continuous and nondecreasing mapping such that

$$
p(f(x), f(y)) \leq p(x, y)-\psi(p(x, y))
$$

for $x \geq y$ where $\psi:[0, \infty) \longrightarrow[0, \infty)$ is a continuous and nondecreasing function such that $\psi(t)>0$ for $t>0, \psi(0)=0$ and $\lim _{t \rightarrow \infty} \psi(t)=\infty$. If there exists $x_{0} \in X$ with $x_{0} \leq f\left(x_{0}\right)$, then $f$ has a fixed point.

proof. If $f\left(x_{0}\right)=x_{0}$, then the proof is finished. Suppose that $x_{0}<f\left(x_{0}\right)$. Since $x_{0}<f\left(x_{0}\right)$ and $f$ is a nondecreasing function, we obtain by induction that

$$
x_{0} \leq f\left(x_{0}\right) \leq f^{2}\left(x_{0}\right) \leq f^{3}\left(x_{0}\right) \leq \ldots \leq f^{n}\left(x_{0}\right) \leq f^{n+1}\left(x_{0}\right) \leq \ldots
$$

Put $x_{n+1}=f\left(x_{n}\right)$. Then for each integer $n \geq 1$, from 2.1 and, as the elements $x_{n}$ and $x_{n+1}$ are comparable, we get

$$
p\left(x_{n+1}, x_{n}\right)=p\left(f\left(x_{n}\right), f\left(x_{n-1}\right)\right) \leq p\left(x_{n}, x_{n-1}\right)-\psi\left(p\left(x_{n}, x_{n-1}\right)\right) .
$$


If there exists $n_{0} \in \mathbb{N}$ such that $p\left(x_{n_{0}}, x_{n_{0}-1}\right)=0$, then

$$
x_{n_{0}}=f\left(x_{n_{0}-1}\right)=x_{n_{0}-1},
$$

and $x_{n_{0}-1}$ is a fixed point and the proof is finished.

Now, suppose that $p\left(x_{n+1}, x_{n}\right) \neq 0$ for all $n \in \mathbb{N}$. Then by 2.1 and our assumptions about $\psi$

$$
p\left(x_{n+1}, x_{n}\right)=p\left(x_{n}, x_{n-1}\right)-\psi\left(p\left(x_{n}, x_{n-1}\right)\right)<p\left(x_{n}, x_{n-1}\right) .
$$

Put $\rho_{n}=p\left(x_{n+1}, x_{n}\right)$. Then we have

$$
\rho_{n} \leq \rho_{n-1}-\psi\left(\rho_{n-1}\right) \leq \rho_{n-1} .
$$

Therefore $\left\{\rho_{n}\right\}$ is a nonnegative nonincreasing sequence and hence possesses a limit $\rho^{*}$ by 2.2, when $n \rightarrow \infty$ we have

$$
\rho^{*} \leq \rho^{*}-\psi\left(\rho^{*}\right) \leq \rho^{*}
$$

and, consequently, $\psi\left(\rho^{*}\right)=0$. So, $\rho^{*}=0$.

In what follows we will show that $\left\{x_{n}\right\}$ is a Couchy sequence. Fix $\varepsilon>0$. As $p_{n}=p\left(x_{n+1}, x_{n}\right) \rightarrow 0$, there exists $n_{0} \in \mathbb{N}$ such that

$$
p\left(x_{n_{0}+1}, x_{n_{0}}\right) \leq \min \left\{\frac{\varepsilon}{2}, \psi\left(\frac{\varepsilon}{2}\right)\right\} .
$$

Note that $\psi\left(\frac{\varepsilon}{2}\right)>0$. We claim that

$$
f\left(\overline{B\left(x_{n_{0}}, \varepsilon\right)} \cap\left\{y \in X: y \geq x_{n_{0}}\right\}\right) \subset \overline{B\left(x_{n_{0}}, \varepsilon\right)} .
$$

Let $z \in \overline{B\left(x_{n_{0}}, \varepsilon\right)} \cap\left\{y \in X: y \geq x_{n_{0}}\right\}$. Then are two cases:

case 1. $p\left(z, x_{n_{0}}\right) \leq \frac{\varepsilon}{2}$.

In this case, as $z$ and $x_{n_{0}}$ are comparable, we have

$$
\begin{aligned}
p\left(f(z), x_{n_{0}}\right) & \leq p\left(f(z), f\left(x_{n_{0}}\right)\right)+p\left(f\left(x_{n_{0}}\right), x_{n_{0}}\right) \\
& =p\left(f(z), f\left(x_{n_{0}}\right)\right)+p\left(x_{n_{0}+1}, x_{n_{0}}\right) \\
& \leq p\left(z, x_{n_{0}}\right)-\psi\left(p\left(z, x_{n_{o}}\right)\right)+p\left(x_{n_{0}+1} x_{n_{0}}\right) \\
& \leq p\left(z, x_{n_{0}}\right)+p\left(x_{n_{0}+1} x_{n_{0}}\right) \leq \frac{\varepsilon}{2}+\frac{\varepsilon}{2}=\varepsilon .
\end{aligned}
$$

case 2. $\frac{\varepsilon}{2}<p\left(z, x_{n_{0}}\right) \leq \varepsilon$.

In this case, as $\psi$ is nondecreasing,

$$
\psi\left(p\left(z, x_{n_{o}}\right)\right) \geq \psi\left(\frac{\varepsilon}{2}\right) .
$$


Therefore from 2.3 we have

$$
\begin{aligned}
p\left(f(z), x_{n_{0}}\right) & \leq p\left(f(z), f\left(x_{n_{0}}\right)\right)+p\left(f\left(x_{n_{0}}\right), x_{n_{0}}\right) \\
& =p\left(f(z), f\left(x_{n_{0}}\right)\right)+p\left(x_{n_{0}+1}, x_{n_{0}}\right) \\
& \leq p\left(z, x_{n_{0}}\right)-\psi\left(p\left(z, x_{n_{o}}\right)\right)+p\left(x_{n_{0}+1} x_{n_{0}}\right) \\
& \leq p\left(z, x_{n_{0}}\right)-\psi\left(\frac{\varepsilon}{2}\right)+\psi\left(\frac{\varepsilon}{2}\right) \leq p\left(z, x_{n_{0}}\right) \leq \varepsilon .
\end{aligned}
$$

This proves the claim.

$$
\begin{aligned}
& \text { As } x_{n_{0}+1} \in \overline{B\left(x_{n_{0}}, \varepsilon\right)} \cap\left\{y \in X: y \geq x_{n_{0}}\right\} \text {, we have } \\
& f\left(x_{n_{0}+1}\right)=x_{n_{0}+2} \in \overline{B\left(x_{n_{0}}, \varepsilon\right)} \cap\left\{y \in X: y \geq x_{n_{0}}\right\}
\end{aligned}
$$

Repeating this process, yields that $x_{n} \in \overline{B\left(x_{n_{0}}, \varepsilon\right)}$ for $n \geq n_{0}$. Since $E$ is arbitrary, $\left\{x_{n}\right\}$ is a Cauchy sequence. Then there exists $z \in X$ such that $\lim _{n \rightarrow \infty} x_{n}=z$.

The continuity of $f$ implies that $z$ is a fixed point. This, the proof is complete.

Theorem 2.2. Let $(X, \leq)$ be a partially ordered set and suppose that there exists a metric $d$ in $X$ such that $(X, d)$ is a complete metric space and let $p$ be a $w$-distance on $X$. Assume that $X$ Satisfies:

If $\left\{x_{n}\right\}$ is a nondecreasing sequence in $X$ such that $x_{n} \rightarrow x$ then

$$
x_{n} \leq x \quad \text { for all } n \in \mathbb{N}
$$

Let $f: X \longrightarrow X$ be a continuous and nondecreasing mapping such that

$$
p(f(x), f(y)) \leq p(x, y)-\psi(p(x, y))
$$

for $x \geq y$ where $\psi:[0, \infty) \longrightarrow[0, \infty)$ is a continuous and nondecreasing function such that $\psi(t)>0$ for $t>0, \psi(0)=0$ and $\lim _{t \rightarrow \infty} \psi(t)=\infty$. If there exists $x_{0} \in X$ with $x_{0} \leq f\left(x_{0}\right)$, then $f$ has a fixed point.

proof. Following the proof of theorem 2.1, we only check that $f(z)=z$. In fact, by using 2.4,

$$
\begin{aligned}
p(f(z), z) & \leq p\left(f(z), f\left(x_{n}\right)\right)+p\left(f\left(x_{n}\right), z\right) \\
& =p\left(z, x_{n}\right)-\psi\left(p\left(z, x_{n}\right)\right)+p\left(x_{n+1}, z\right)
\end{aligned}
$$

and taking limit as $n \rightarrow \infty, p(f(z), z) \leq 0$ and this proves that $p(f(z), z)=0$. Consequently, $f(z)=z$. 


\section{Theorem 2.3. Adding condition}

For $x, y \in X$ there exists $z \in X$ which is comparable to

$$
x \text { and } y
$$

to the hypotheses of theorem 2.1 (resp. theorem 2.2) we obtain uniqueness of the fixed point of $f$.

proof. Suppose that there exist $z, y \in X$ which are fixed points. We distinguish two cases:

Case 1. If $y$ is comparable to $z$, then $f^{n}(y)=y$ is comparable to $f^{n}(z)=z$ for $n=0,1,2, \ldots$, and

$$
\begin{aligned}
p(z, y) & =p\left(f^{n}(z), f^{n}(y)\right) \\
& \leq p\left(f^{n-1}(z), f^{n-1}(y)\right)-\psi\left(p\left(f^{n-1}(z), f^{n-1}(y)\right)\right) \\
& \leq p(z, y)-\psi(p(z, y) \leq p(z, y) .
\end{aligned}
$$

Consequently, $\psi(p(z, y))=0$ and this gives us that $p(z, y)=0$.

Case 2. If $y$ is not comparable to $z$, then there exists $x \in X$ comparable to $y$ and $z$. Monotonicity implies that $f^{n}(x)$ is comparable to $f^{n}(y)=y$ and $f^{n}(z)=z$ for $n=0,1,2, \ldots$ Moreover,

$$
\begin{aligned}
p\left(z, f^{n}(x)\right) & =p\left(f^{n}(z), f^{n}(x)\right) \\
\leq p & \left(f^{n-1}(z), f^{n-1}(x)\right)-\psi\left(p\left(f^{n-1}(z), f^{n-1}(x)\right)\right) \\
\leq & p\left(f^{n-1}(z), f^{n-1}(x)\right) .
\end{aligned}
$$

Consequently, $p\left(z, f^{n}(x)\right)=p\left(f^{n}(z), f^{n}(x)\right)$ is a nonnegative nonincreasing sequence and hence possesses limit $\gamma$.

From the last inequality we can obtain $\gamma \leq \gamma-\psi(\gamma) \leq \gamma$ and hence $\psi(\gamma)=0$, so $\gamma=0$. Analogously, it can be proved that

$$
\lim _{n \rightarrow \infty} p\left(y, f^{n}(x)\right)=0 .
$$

Finally,

$$
p(z, y) \leq p\left(z, f^{n}(x)\right)+p\left(f^{n}(x), y\right)
$$

and taking limit we have $p(z, y)=0$.

Now, we deal with nonincreasing functions. 
Theorem 2.4. Let $(X, \leq)$ be a partially ordered set varifying 2.5 and suppose that there exists a metric $d$ in $X$ such that $(X, d)$ is a complete metric space and $p$ is a $w$-distance on $X$.

Let $f: X \longrightarrow X$ be a nonincreasing function such that

$$
p(f(x), f(y)) \leq p(x, y)-\psi(p(x, y))
$$

for $x \geq y$ where $\psi:[0, \infty) \longrightarrow[0, \infty)$ satisfies the conditions appearing in theorem 2.2. Suppose also that either $f$ is a continuous,

or

$X$ is such that if $\left(x_{n}\right) \rightarrow x$ is a sequence in $X$ whose consecutive terms are comparable,

then there exists a subsequence $\left\{x_{n_{k}}\right\}$ of $\left\{x_{n}\right\}$ such that every term is comparable to the limit $x$.

If there exists $x_{0} \in X$ with $x_{0} \leq f\left(x_{0}\right)$ or $x_{0} \geq f\left(x_{0}\right)$ then $f$ has a unique fixed point.

proof. If $f\left(x_{0}\right)=x_{0}$, then the existence of a fixed point is proved. Suppose that $f\left(x_{0}\right) \neq x_{0}$. Following the lines of the proof of theorem 2.1, we obtain that $\left\{f^{n}\left(x_{0}\right)\right\}$ is a convergent sequence in $X$.

Indeed, by our assumption $f^{n+1}\left(x_{0}\right)$ and $f^{n}\left(x_{0}\right)$ are comparable, for every $n=$ $0,1,2, \ldots$ and therefore, by induction

$$
\begin{aligned}
p\left(f^{n+1}\left(x_{0}\right), f^{n}\left(x_{0}\right)\right) \leq & p\left(f^{n}\left(x_{0}\right), f^{n-1}\left(x_{0}\right)\right)-\psi\left(p\left(f^{n}\left(x_{0}\right), f^{n-1}\left(x_{0}\right)\right)\right) \\
& \leq p\left(f^{n}\left(x_{0}\right), f^{n-1}\left(x_{0}\right)\right) .
\end{aligned}
$$

This proves that the sequence $\left\{p\left(f^{n+1}(x), f^{n}\left(x_{0}\right)\right)\right\}$ is a nonnegative nonincreasing sequence with limit $\rho^{*}$. Using the same argument that in theorem 2.1, we prove that $\rho^{*}=0$.

The same reasoning that in theorem 2.1 gives us that $\left\{f^{n}\left(x_{0}\right)\right\}$ is a cauchy sequence and consequently $\left\{f^{n}\left(x_{0}\right)\right\}$ is convergent to some $z \in X$.

In the case that $f$ is continuous it is easily seen that $z$ is a fixed point.

Suppose that condition 2.6 holds. Since $f$ is nonincreasing $\left\{f^{n}\left(x_{0}\right)\right\}$ is not necessarily monotone, but it is a convergent sequence with comparable consecutive terms. Then, by condition 2.5, there exists a subsequence $\left\{f^{n_{k}}\left(x_{0}\right)\right\}$ consisting of terms which are comparable to the limit $z$. Hence, for $k \in \mathbb{N}$

$$
\begin{aligned}
& p(f(z), z) \leq p\left(f(z), f^{n_{k}+1}\left(x_{0}\right)\right)+p\left(f^{n_{k}+1}\left(x_{0}\right), z\right) \\
& \leq p\left(z, f^{n_{k}}\left(x_{0}\right)\right)-\psi\left(p\left(z, f^{n_{k}}\left(x_{0}\right)\right)\right)+p\left(f^{n_{k}+1}\left(x_{0}\right), z\right)
\end{aligned}
$$




$$
\leq p\left(z, f^{n_{k}}\left(x_{0}\right)\right)+p\left(f^{n_{k}+1}\left(x_{0}\right), z\right)
$$

Taking limit as $k \rightarrow \infty$, we obtain that $p(f(z), z)=0$. The uniqueness of the fixed point is proved as in theorem 2.3.

Finally, we show that the monotonicity of $f$ is not essential for the existence of a fixed point, we replace this condition by the preservation of comparable elements which is trivially verified if $X$ is totally ordered.

Theorem 2.5. Let $(X, \leq)$ be a partially ordered set and suppose that 2.5 holds and that there exists a metric $d$ in $X$ such that $(X, d)$ is a complete metric space and let $p$ be a $w$-distance on $X$. Let $f: X \longrightarrow X$ be such that $f$ maps comparable elements into comparable elements, that is, for $x, y \in X$,

$$
x \leq y \quad \Longrightarrow \quad f(x) \leq f(y) \quad \text { or } \quad f(x) \geq f(y)
$$

and such that for $x, y \in X$ with $x \geq y$

$$
p(f(x), f(y)) \leq p(x, y)-\psi(p(x, y))
$$

where $\psi:[0, \infty) \longrightarrow[0, \infty)$ satisfies the conditions appearing in theorem 2.1. Suppose that either $f$ is continuous or $X$ is such that condition 2.6 holds. If there exists $x_{0} \in X$ with $x_{0}$ comparable to $f\left(x_{0}\right)$, then $f$ has a unique fixed point $\bar{x}$. Moreover, for $x \in X, \lim _{n \rightarrow \infty} f^{n}(x)=\bar{x}$.

proof. Since $x_{0} \in X$ is comparable to $f\left(x_{0}\right)$, then $f^{n+1}\left(x_{0}\right)$ and $f^{n}\left(x_{0}\right)$ are comparable for $n=0,1,2, \ldots$ the argument exposed in the proof of theorem 2.4 is valid.

\section{REFERENCES}

[1] E.A. Bahri and L. P. Lions, Generalized Distance and fixed Point Theorems for Weakly Contractive Mapping, (2013), 161-164.

[2] Harjani, K. Sadrangani, Fixed Point Theorems for Weakly Contractive Mappings in Partially Ordered Sets, in Ninlinear Analysis 71 (2009) 3403-3410.

[3] Jeong sheok Ume, Nonlinear Existence Theorems for Generalized Distance on Complete Metric Spaces, (2010) 21 pages.

[4] O. Kada, T. Suzuki and W.Takahashi, Nonconvex Minimization Theorems and Fixed Point Theorems in Complete Metric Spaces, Math. Japonica, 44, (1996) 381-391. 
[5] Mohammad Imdad and Fayyaz Rouzkard, Fixed Point Theorems in Ordered Metric Spaces Via w-distances, Fixed Point Theory and Applications (2012).

[6] Mujahid Abbas. Dejan Ilic and Muhammad Ali khan, Coupled Coincidence Point and Coupled Common Fixed Point Theorems in Partially Ordered Metric spaces with $w$-Distance, (2010) 11 pages.

[7] A. Razani, Z. Mazluminezhad and M. Boujary, A Fixed Point Theorem for w-Distance, (2009)114-117.

[8] Fayyaz Rouzkard, Mohammad Imdad and Dhananjay Gopal, Some Existence and Uniqueness Theorems on Ordered Metric Spaces Via Generalized Distances.

[9] T. Suzuki, Fixed Point Theorems in Complete Metric Spaces, in Nonlinear Analysis and Convex Analysis (W. Takahashi, Ed), Vol. 939, pp. 173-182, RIMS, Kokyurku, (1996).

[10] W. Takahashi, , Nonlinear Function Analysis, Yokohama publishere, (2000).

Received: August 5, 2014; Published: October 23, 2014 